\title{
A Multimodal Navigation System for Interventional MRI
}

\author{
K. Kansy ${ }^{1}$, A. Schmitgen ${ }^{1,2}$, M. Bublat ${ }^{1,2}$, G. Grunst ${ }^{1}$, M. Jungmann ${ }^{1}$, \\ P. Wisskirchen ${ }^{1}$, M. Moche ${ }^{3}$, G. Strauss ${ }^{3}$, C. Trantakis ${ }^{3}$, and T. Kahn ${ }^{3}$ \\ ${ }^{1}$ GMD - German National Research Center for Information Technology, \\ D-53754 Sankt Augustin, Germany \\ kansy@gmd.de, http://fit.gmd.de/results/localite/ \\ 2 LOCALITE GmbH, Graurheindorfer Str. 69, D-53111 Bonn, Germany \\ 3 Universitätsklinikum Leipzig, Liebigstrasse 20a, D-04103 Leipzig, Germany
}

\begin{abstract}
Medical imaging permits high precision minimally invasive interventions even in very delicate situations but leads to complex medical procedures not easily applied in a hospital setting. The LOCALITE Navigator is an interactive navigation system that comprises state-of-the art registration and visualization of multimodal medical images for minimally invasive interventions with an interventional magnetic resonance imaging (iMRI) system. The focus is on an intuitive and simple user interface that can be handled by the medical personnel themselves without relying on computer experts. The system has got a CE certificate in 2001 and is used by all GE SIGNA SP installations in Germany.
\end{abstract}

\section{Design of the LOCALITE Navigator}

The LOCALITE Navigator was designed to improve the handling of interventional Magnetic Resonance Imaging systems, specifically the SIGNA SP (0.5 T) of General Electric Medical Systems, Milwaukee, WI, USA. It tackles the following problems:

Image Quality. Due to the bad resolution (time and space) of real-time images, (some) experts regard them near to useless. LOCALITE complements them with corresponding slices calculated from intra-operative 3D-data (a similar approach is used by 3D-slicer at Surgical Planning Lab at Brigham and Women's Hospital [1]). The calculated slices are an excellent means for interpreting and understanding the low quality real-time slices.

Interactivity. The SIGNA SP is equipped with a camera-based locator system (Flashpoint, IGT, Boulder, CO, USA), which precisely measures position and orientation of a hand piece at a rate of about $10 \mathrm{~Hz}$. The real-time MR images are scanned in any plane relative to the tip of device however with a delay of several seconds. The calculated planes of LOCALITE can follow the speed of the locator device.

Orientation. In real-time mode, the SIGNA SP only generates single 2D slices. We have developed an abstract scene containing just the elements necessary for moving the device: markers for planned entry and target points and a 
virtual device showing the current position and orientation of the real device. This simple scene gives the surgeon sufficient orientation to perform positioning in seconds rather than minutes or even longer in delicate cases.

Multimodal Visualisation (Image Fusion). In addition to the interventional images, pre-operative data sets $(\mathrm{CT}, \mathrm{MR}, \mathrm{fMR})$ may be required during the intervention. LOCALITE offers different presentation options for two data sets. Two data sets can be overlaid with transparency. Sliders allow a dynamic setting of the transparency levels. In this mode, the bright structures in both data sets will be shown. For typical mixes, predefined overlay modes can be selected by buttons, e.g., emphasizing a specific structure (bone) by colour or instantaneous switching to one data set.

Registration. Different data sets can only be displayed consistently within one scene after geometrical alignment of coordinate systems (registration). LOCALITE includes a robust registration mechanism starting with a rough landmarkbased registration followed by automatic registration based on mutual information which yields optimal precision for MR and CT. In the case of T2-weighted images of functional MR (fMR), this registration fails. Using a co-registered MR rather than the fMR data set (both scanned in one session with patient in a fixed position) solves the problem. The location of functional areas can now be related to the current position of the medical device.

\section{Discussion}

The LOCALITE Navigator is based on the LOCALITE system [2] and includes the functionality needed for a multimodal navigation system for iMRI and only this functionality. It has been developed in close cooperation with clinicians and is used inside and outside of a university setting by all SIGNA SP installations in Germany. Currently, we are working on a transfer of concepts to the imaging modality ultrasound, which is ubiquitously available and opens a wider market.

LOCALITE Navigator is programmed in Java on a standard PC with Windows NT 4.0 and graphics card supporting OpenGL.

\section{References}

1. David Gering (1999) A System for Surgical Planning and Guidance using Image Fusion and Interventional MR. MIT Master's Thesis.

2. Klaus Kansy et al. (1999) LOCALITE - a Frameless Neuronavigation System for Interventional Magnetic Resonance Imaging Systems. In: C. Taylor, A. Colchester (eds) Medical Image Computing and Computer-Assisted Intervention - MICCAI'99. Berlin: Springer (1999) 832-841 